\title{
Posttraumatic Rupture of Branch Pseudoaneurysm of Deep Femoral artery: A Rare Case
}

\author{
Rajendra Prasad ${ }^{1} \quad$ Luv Luthra $^{1} \quad$ Adharsh Maruthu $^{1}$ \\ 1Department of Endovascular Surgery, MS Ramaiah Medical \\ College, Bengaluru, Karnataka, India \\ Address for correspondence Luv Luthra, MS, Department of \\ Endovascular Surgery, MS Ramaiah Medical College, Bengaluru \\ 560054, Karnataka, India (e-mail: drluv.luthra@gmail.com).
}

Int J Recent Surg Med Sci 2019;5:58-61

\begin{abstract}
Introduction Traumatic pseudoaneurysms of deep femoral artery (DFA) are usually seen secondary to sports injuries, postendovascular procedures, trauma to thigh, or after orthopaedic interventions for femur fractures. They usually present as either a pulsatile mass or even as thigh compartment syndrome if not diagnosed early.

Case Report We present a case of a 65-year-old male who was referred for thigh swelling with severe anemia. On angiographic evaluation, patient was diagnosed to have pseudoaneurysm of DFA branch with hematoma in the thigh. Patient underwent an emergency surgery in view of hemodyanamic instability and ligation of ruptured

Keywords

- deep femoral artery

- ligation

- pseudoaneurysm

- rupture DFA branch pseudoaneurysm.

Conclusion Pseudoaneurysms of the DFA are rare entity and can be asymptomatic or may present with active bleeding in the compartment on rupture. Both surgical and endovascular treatments are available options and mainly depend on the hemodyanamic condition of the patient.
\end{abstract}

\section{Introduction}

Traumatic pseudoaneurysms of deep femoral artery (DFA; profunda artery) are usually seen secondary to sports injuries, postendovascular procedures, trauma to thigh, or after orthopaedic interventions for femur fractures. They usually present as either a pulsatile mass or even as thigh compartment syndrome if not diagnosed early. ${ }^{1}$

Majority of patients present with pulsatile mass but huge pseudoaneurysms can clinically manifest with compression symptoms of pain and neurological deficits. In case of ruptured pseudoaneurysms patients may present with life-threatening bleeding and hypovolemic shock. ${ }^{2}$

Pseudoaneurysm is one of the most serious complications after femoral trochanteric fracture surgery. The occurrence of pseudoaneurysm in the branch of DFA after surgical repair of trochanteric fracture is relatively rare and also presents with nonspecific signs of pain, subcutaneous bleeding, unexplained anemia, and swelling of the thigh. ${ }^{3}$

We present a case of a 65 -year-old male with severe anemia and ruptured DFA branch pseudoaneurysm.

\section{Case Report}

A 65-year-old male was referred to vascular surgery with a sudden onset swelling in the right thigh since 3 days post a fall while walking at his home. Patient had undergone a right trochanteric femur fracture surgery 2 months back and now was admitted with right-thigh swelling.

On examination, the patient had a tensed swelling in the thigh with ecchymotic patches and edema extending up to the foot. Ankle toe movements were restricted with absent popliteal and distal pulses. Patient was severely anemic (hemoglobin $=5.3$ ), in spite of undergoing three blood transfusions. Local site sonography showed an expanding hematoma in the anterior, lateral, and medial aspect of right thigh with collection of around $1,400 \mathrm{cc}$ in the compartment (-Figs. 1-3).

Patient was taken up for emergency exploration after confirmation of pseudoaneurysm on computed tomography angiography (CTA) and deteriorating hemodyanamic condition on the same day. Written informed consent was taken, and the risks associated with surgery with risk of limb loss were explained. Intraoperatively, around $1 \mathrm{~L}$ of clots 


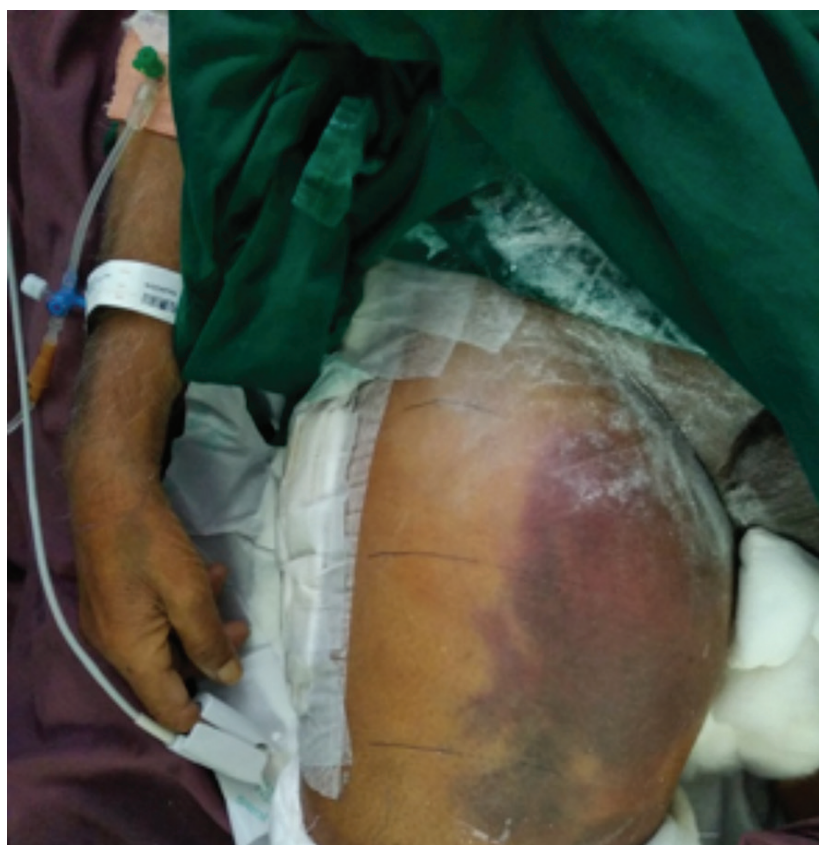

Fig. 1 Tensed swelling in the right thigh with edema.

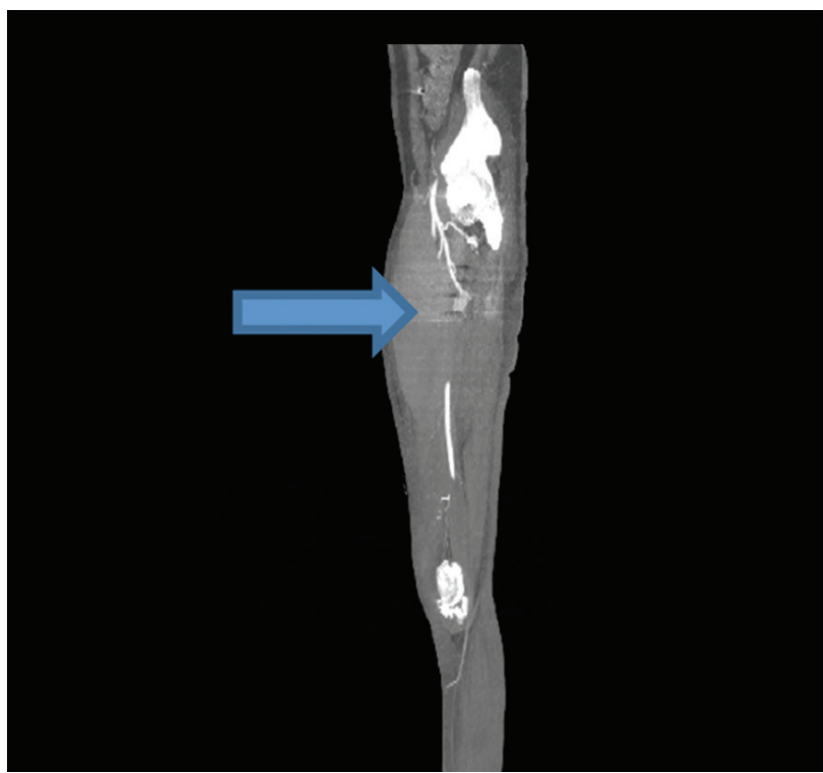

Fig. 3 CT angiography showed a pseudoaneurysm of the branch of deep femoral artery. Arrow shows the part of the aneurysm/sac arising from the branch of deep femoral artery with nonopacification of the mid superficial femoral artery. CT, computed tomography.

was evacuated, and active bleeding was noted in the lateral aspect of thigh from a ruptured pseudoaneurysm. Proximal and distal control of common femoral artery (CFA), DFA, and superficial femoral artery (SFA) were taken. The ruptured pseudoaneurysm sac was excised and branch of DFA ligated.

Implant was removed by orthopaedic surgeon and revision surgery was done. Patient recovered well with no neurological, vascular deficit at the time of discharge.

Intraoperative images are shown in -Figs. 4-7.

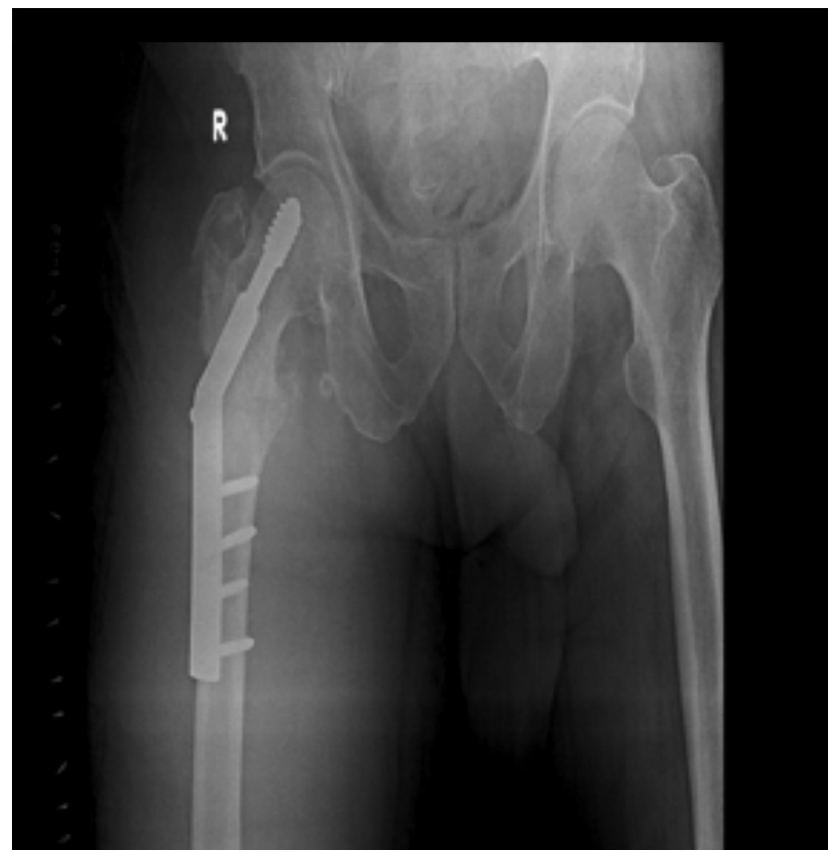

Fig. 2 X-Ray of the right leg shows right femur fixed with dynamic hip screw and a plate with one of the screws protruding the bone.

\section{Discussion}

DFA injuries may be overlooked due to delayed presentation and also because distal pulses are usually present. Accurate diagnosis is difficult as this artery is located deep in the thigh. ${ }^{4}$ Pseudoaneurysms occur due to disruption in continuity of arterial wall caused by trauma, surgery, or endovascular procedures. ${ }^{5}$ The intermedullary nail used to treat trochanteric fractures can cause pseudoaneurysm after dislocation from the bone. ${ }^{6}$

The DFA artery runs along inside of the femur, and can be easily damaged by drilling under the hip joint on adduction and internal rotation. ${ }^{7}$ Pseudoaneurysms typically present late and signs, such as persistent hip pain, thigh swelling, and the presence of a pulsatile mass and unexplained anemia, may suggest the diagnosis. ${ }^{8}$ Ruptured pseudoaneurysms can be life-threatening and lead to hemorrhagic shock and even death if not encountered immediately.

Doppler and CT angiography are investigations of choice for identifying aneurysms, to remove sensitivity and specificity in the line. Multidetector CTA enables three-dimensional reformatting of the lower limb vasculature. It is a quick and noninvasive method, with high sensitivity (90-95\%) and specificity (98-100\%) for detecting arterial injury after trauma. ${ }^{9}$ Experience in treatment of DFA pseudoaneurysms is limited.

Symptomatic pseudoaneurysms are always treated whereas surgical intervention for asymptomatic pseudoaneurysms is controversial. The majority of small pseudoaneurysms $(<2-3 \mathrm{~cm}$ in diameter) usually thrombose spontaneously within 4 weeks. ${ }^{10,11}$ Small, asymptomatic PSAs should be observed with serial scans and treated only if they enlarge or do not resolve, or become symptomatic. ${ }^{12}$ False 


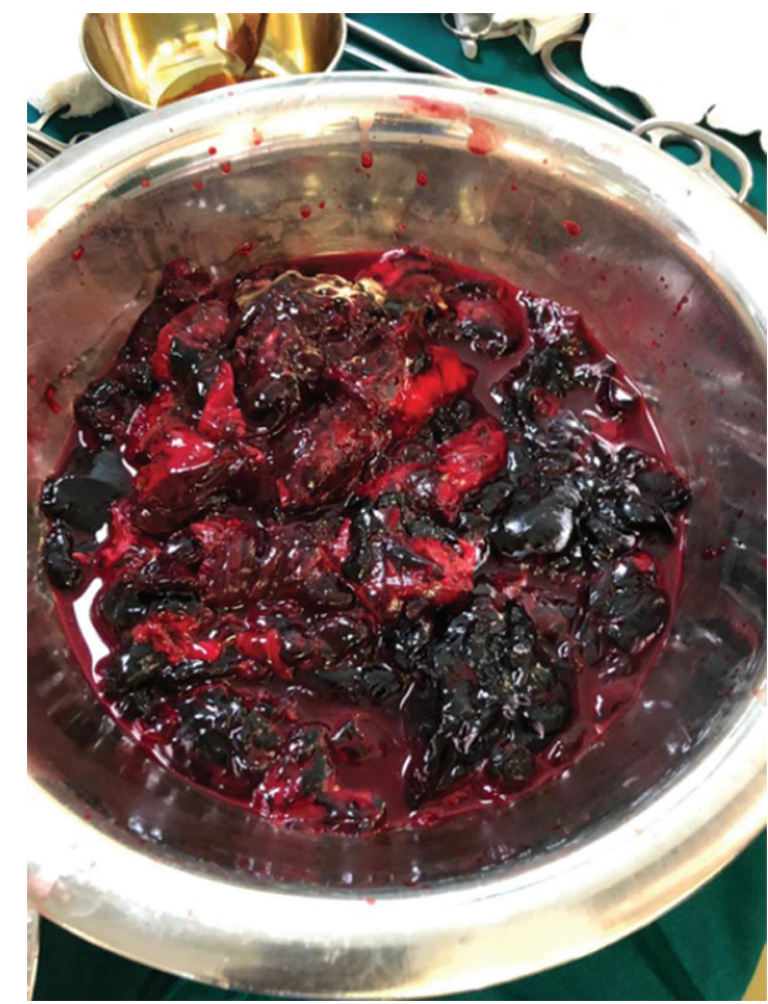

Fig. 4 Evacuated clots.

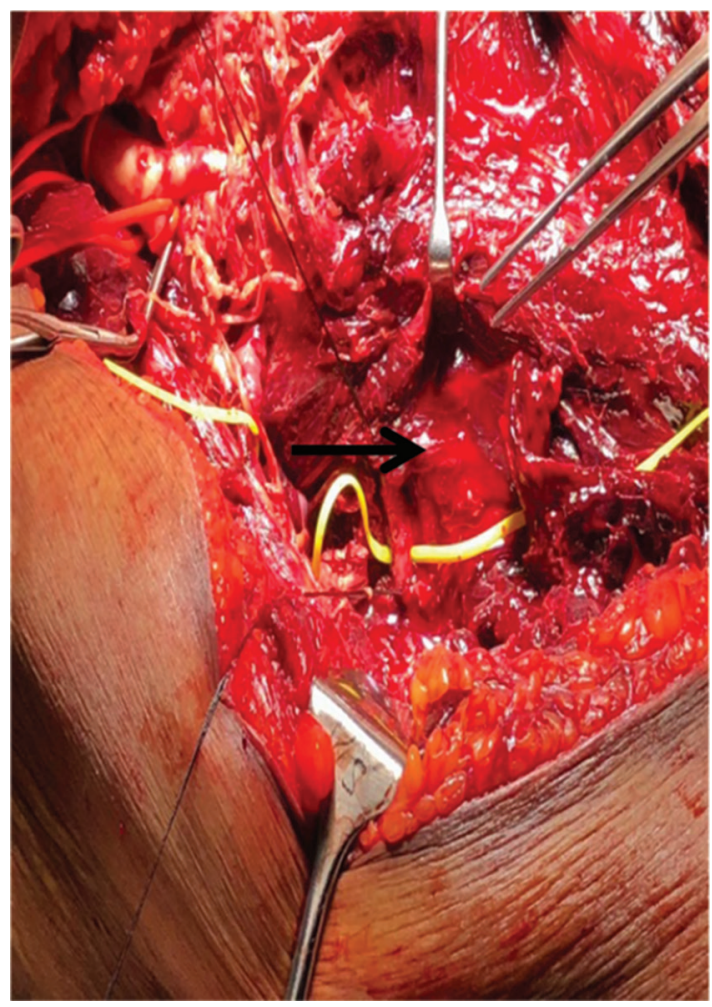

Fig. 5 Pseudoaneurysm sac (arrow).

aneurysm can obliterate spontaneously, especially when smaller than one inch and with a small neck $(<10 \mathrm{~mm}){ }^{13}$

Current therapeutic approaches include open surgical repair, ultrasound-guided compression, ultrasound-guided thrombin injection, and endovascular repair using coil

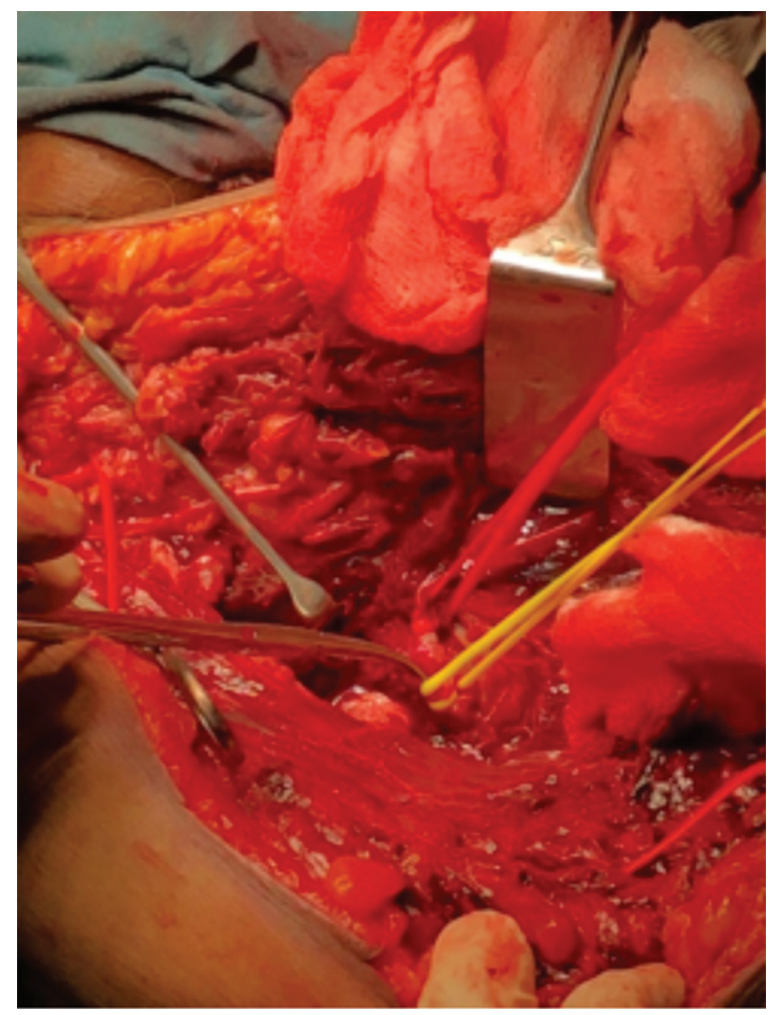

Fig. 6 Control of deep femoral artery and deep femoral artery.

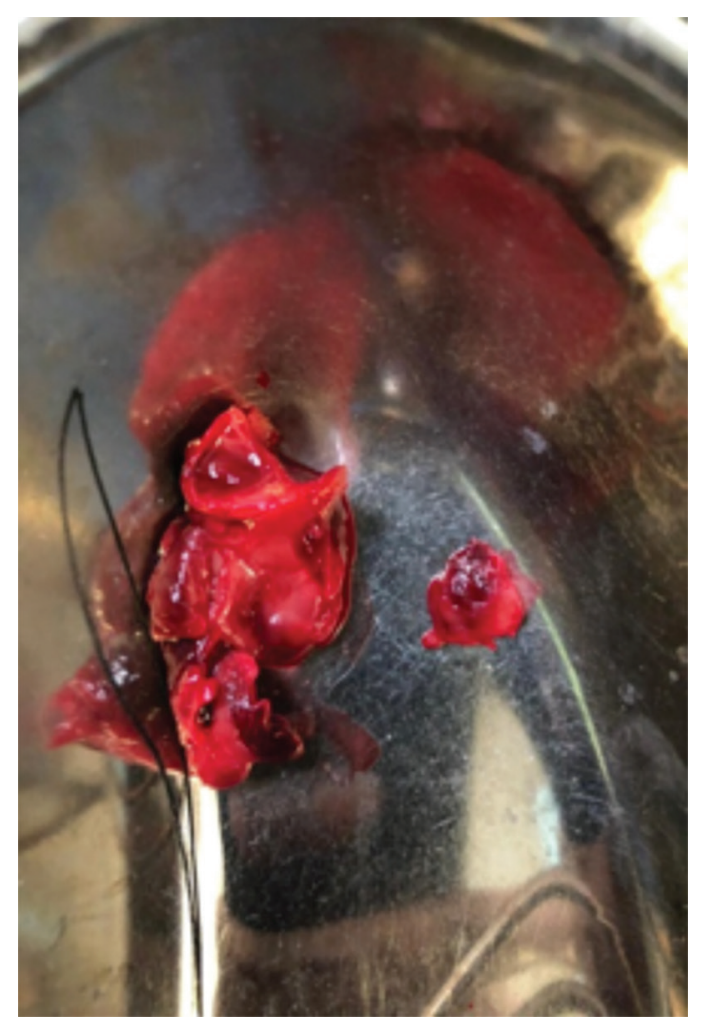

Fig. 7 Removed pseudoaneurysm sac.

embolization or stent-graft insertion. Open surgery is the treatment of choice in case of compressive neuropathy, limb ischaemia, distal embolization, skin necrosis, infection, or the risk of rupture. ${ }^{14}$ The incidence of postoperative complications may be as high as $20 \%$ with up to a $3 \%$ mortality 
rate. ${ }^{15}$ The anteromedial approach to whole trunk of the DFA is now the standard one for of most procedures DFA reconstruction.

\section{Conclusion}

In our experience, it was the first case of ruptured pseudoaneurysm arising from DFA and patient was in hemodyanamic shock due to persistent blood loss. Excision and ligation of the aneurysm were the best possible treatments in this situation and patient recovered well with no threat to the limb. Angiography is the investigation of choice in cases where rupture of pseudoaneurysms is suspected.

\section{Conflict of Interest}

None declared.

\section{References}

1 Lewis DR, Bullbulia RA, Murphy P, et al. Vascular surgical intervention for complications of cardiovascular radiology: 13 years' experience in a single centre. Ann R Coll Surg Engl 1999;81(1):23-26

2 Doiz E, Garrido F, Conejero R, García P, Fernández E. Acute pseudoaneurysm of the femoral artery after repeated trauma in full-contact karate practice. Br J Sports Med 2008;42(12):1004-1005

3 Kinoshita H, Hashimoto M, Hirayama J, et al. Huge pseudoaneurysm of the femoral artery after internal fixation of femoral trochanteric fracture. Case Rep Orthop 2018;2018:3182643

4 Khoshnevis J, Sobhiyeh MR, Fallah Zavareh M. Deep femoral artery branch pseudoaneurysm after orthopedic procedure requiring surgical treatment: a case report. Trauma Mon 2012;17(2):305-308
5 Schwartz LB, Clark ET, Gewertz BL. Anastomotic and other pseudoaneurysms. In: Rutherford RB, ed. Vascular Surgery. 5th ed. Philadelphia, PA: Saunders; 2000: 752-763

6 Chan WS-W, Kong S-W, Sun K-W, Tsang P-K, Chow H-L. Pseudoaneurysm and intramuscular haematoma after dynamic hip screw fixation for intertrochanteric femoral fracture: a case report. J Orthop Surg (Hong Kong) 2010;18(2):244-247

7 Yang KH, Park HW, Park SJ. Pseudoaneurysm of the superficial femoral artery after closed hip nailing with a Gamma nail: report of a case. J Orthop Trauma 2002;16(2):124-127

8 Hanna GB, Holdsworth RJ, McCollum PT. Profunda femoris artery pseudoaneurysm following orthopaedic procedures. Injury 1994;25(7):477-479

9 Miller-Thomas MM, West OC, Cohen AM. Diagnosing traumatic arterial injury in the extremities with CT angiography: pearls and pitfalls. Radiographics 2005;25(Suppl 1):S133-S142

10 Middleton WD, Dasyam A, Teefey SA. Diagnosis and treatment of iatrogenic femoral artery pseudoaneurysms. Ultrasound $Q$ 2005;21(1):3-17

11 Toursarkissian B, Allen BT, Petrinec D, et al. Spontaneous closure of selected iatrogenic pseudoaneurysms and arteriovenous fistulae. J Vasc Surg 1997;25(5):803-808, discussion 808-809

12 Saad NE, Saad WE, Davies MG, Waldman DL, Fultz PJ, Rubens DJ. Pseudoaneurysms and the role of minimally invasive techniques in their management. Radiographics 2005;25(Suppl 1):S173-S189

13 Ritchie ED, Haverkamp D, Schiphorst TJMJ, Bosscha K. False aneurysm of the profunda femoris artery, a rare complication of a proximal femoral fracture. Acta Orthop Belg 2007;73(4):530-532

14 O'Sullivan GJ, Ray SA, Lewis JS, et al. A review of alternative approaches in the management of iatrogenic femoral pseudoaneurysms. Ann R Coll Surg Engl 1999;81(4):226-234

15 Sedwitz MM, Hye RJ, Stabile BE. The changing epidemiology of pseudoaneurysm. Therapeutic implications. Arch Surg 1988;123(4):473-476 